\title{
Towards habitable Earths with EUCLID and WFIRST
}

\author{
Jean-Philippe Beaulieu ${ }^{1,2}$, David P. Bennett ${ }^{3}$, Eamonn Kerins ${ }^{4}$ and \\ Matthew Penny ${ }^{4}$ \\ ${ }^{1}$ Institut d'Astrophysique de Paris, 98bis Boulevard Arago, 75014 PARIS, France \\ ${ }^{2}$ University College London, Gower street, London WC1E 6BT, UK \\ email: beaulieu@iap.fr \\ ${ }^{3}$ Department of Physics, University of Notre Dame, Notre Dame, Indiana 46556, USA \\ email: bennett@nd.edu \\ ${ }^{4}$ Jodrell Bank Centre for Astrophysics, University of Manchester M13 9PL, UK \\ email: Eamonn.Kerins@manchester .ac.uk
}

\begin{abstract}
The discovery of extrasolar planets is arguably the most exciting development in astrophysics during the past 15 years, rivalled only by the detection of dark energy. Two projects are now at the intersection of the two communities of exoplanet scientists and cosmologists: EUCLID, proposed as an ESA M-class mission; and WFIRST, the top-ranked large space mission for the next decade by the Astro 2010 Decadal Survey report. The missions are to have several important science programs: a dark energy survey using weak lensing, baryon acoustic oscillations, Type Ia supernova, a survey of exoplanetary architectures using microlensing, and different surveys. The WFIRST and EUCLID microlensing planet search programs will provide a statistical census of exoplanets with masses greater than the mass of Mars and orbital separations ranging from $0.5 \mathrm{AU}$ outwards, including free-floating planets. This will include analogs of all Solar System planets except for Mercury, as well as most types of planets predicted by planet formation theories. In combination with Kepler's census of planets in shorter period orbits, EUCLID and WFIRST's planet search programs will provide a complete statistical census of the planets that populate our Galaxy. As of today, EUCLID is proposed to ESA as a M class mission (the result of the selection will be known in october 2011). We are presenting here preliminary results about the expected planet yields. WFIRST has just appointed a Science Definition Team.
\end{abstract}

Keywords. planets and satellites: formation, planets and satellites: general, planetary systems, planetary systems: formation

\section{Microlensing today: Super Earths beyond the snow line}

Several different methods have been used to discover exoplanets, including radial velocity, stellar transits, direct imaging, pulsar timing, astrometry, and gravitational microlensing (Mao \& Paczynski 1991; Gould \& Loeb 1992). Microlensing exploits the light deflection effect according to Einstein's theory of general relativity. So far 12 microlensing exoplanets have been published and whilst this number is relatively modest compared with the radial velocity and transit methods, microlensing probes a part of the parameter space (host separation vs. planet mass) not accessible in the medium term to other methods. The mass distribution of microlensing exoplanets has already revealed that cold super-Earths (at or beyond the snow line and with a mass of around 5 to 15 Earth mass) appear to be common (Beaulieu et al. 2006; Gould et al. 2006; Sumi et al. 2010; Cassan et al. 2011). Detections include a scale $1 / 2$ model of our solar system (Gaudi et al. 
2008; Bennett et al. 2010) and several cold Neptunes/Super Earths. It has be shown that the ground-based detection efficiency can, under favourable circumstances, extend down to 1 Earth mass planets (Bennett \& Rhie 1996; Batista et al. 2009). Microlensing has also provided the first measurement of the frequency of ice and gas giants beyond the snow line. The abundance of such systems is about 7 times higher than closer-in planets probed by the Doppler method. This comparison provides strong evidence that most giant planets do not migrate very far (Gould et al. 2010).

Microlensing is currently capable of providing statistics on cool planets of super-Earth mass from the ground. A network of wide-field telescopes, strategically located around the world, could detect planets with mass as low as the Earth. Free-floating planets can also be detected; a significant population of such planets are expected to be ejected during the formation of planetary systems. A wide-field imager network is being implemented now in Chile (OGLE-IV), New Zealand (MOA-2), Tasmania, and the Wise Observatory in Israel. In the near future three additional $1.6 \mathrm{~m}$ telescopes will be deployed as part of the Korean Microlensing network of Telescopes (PI Han). Microlensing is roughly uniformly sensitive to planets orbiting all types of star, as well as white dwarfs, neutron stars, and black holes. In contrast other detection methods are most sensitive to FGK dwarfs and are only now extending to $\mathrm{M}$ dwarfs. Microlensing is therefore an independent and complementary detection method for aiding a comprehensive understanding of the planet formation process. Ground-based microlensing mostly probes exoplanets beyond the snow line, where the favoured core-accretion theory of planet formation predicts a larger number of low-mass exoplanets (Ida and Lin, 2005). The statistics provided by microlensing will enable a critical test of the core accretion model. Exoplanets probed by microlensing are much further away than those probed with other methods and therefore provide an interesting comparison sample with nearby exoplanets. The microlensing road map has been presented in different white papers (ie Gould et al. 2007; Beaulieu et al. 2008; Gaudi et al. 2009; Bennett et al. 2010).

\section{Space based microlensing and Dark Energy}

Ultimately, a comprehensive census of cold planets below Earth masses, including habitable planets, requires a space-based microlensing survey.

Angular resolution is the key to extend sensitivity below a few earth masses Microlensing relies upon the high density of source and lens stars towards the Galactic bulge to gaurantee the stellar alignments needed to generate microlensing events. But this high star density also means that the bulge main sequence source stars are not generally resolved in ground-based images. This means that the precise photometry needed to detect planets of $\leqslant 1 M_{\oplus}$ is not possible from the ground unless the magnification due to the stellar lens is moderately high. This, in turn, implies that ground-based microlensing is only sensitive to terrestrial planets located close to the Einstein ring (at $\sim 2-3 \mathrm{AU}$ ). The full sensitivity to terrestrial planets in all orbits from $0.5 A U$ to free floating comes only from a space-based survey.

Microlensing from space yields precise star and planet parameters

The high angular resolution and stable point-spread-functions available from space enable a space-based microlensing survey to detect most of the planetary host stars. When combined with the microlensing light curve data, this allows a precise determination of the planet and star properties for most events (Bennett et al. 2007a).

The first envisioned project was the Galactic Exoplanet Survey Telescope (GEST) (Bennett \& Rhie 2002), which was a wide-field optical telescope that focused on exoplanets, but also had significant weak lensing and supernova programs when proposed 
to NASA's Midex program. But, the microlensing target fields in the Galactic bulge are most easily observed in the infrared, so the GEST concept was superseded by the wide field infrared Microlensing Planet Finder (MPF) mission concept (Bennett et al. 2007b, 2010). In 2008, the Exoplanet Task Force (ExoPTF) released a report (Lunine et al. 2008) that evaluated all of the current and proposed methods to find and study exoplanets, and expressed strong support for space-based microlensing. Its finding regarding space-based microlensing states that: "Space-based microlensing is the optimal approach to providing a true statistical census of planetary systems in the Galaxy, over a range of likely semi-major axes, and can likely be conducted with a Discovery-class mission." Shortly afterwards the Astro 2010 Decadal Survey ranked as the top priority the WFIRST concept, a wide-field infra-red imager and low resolution spectroscopy on a $1.5 \mathrm{~m}$ telescope aimed at probing dark energy and exoplanet statistics. It "will open up a new frontier of exoplanet studies by monitoring a large sample of stars in the central bulge of the Milky Way for changes in brightness due to microlensing by intervening solar systems. This census, combined with that made by the Kepler mission, will determine how common Earth-like planets are over a wide range of orbital parameters".

We will briefly describe the status of EUCLID and WFIRST in the next two sections:

\subsection{EUCLID}

The use of cosmic shear to probe dark energy was advanced in Europe in 2006 through the DUNE proposal (Refregier et al. 2007), which subsequently developed into EUCLID, based upon a $1.2 \mathrm{~m}$ Korsch telescope. A 3 month microlensing program is part of the additional science of EUCLID, aiming at low mass telluric planets situated around the snow line (described in the EUCLID yellow book and in Beaulieu et al. 2010). The required data for this program is continuous monitoring with a sampling time of around 25 minutes over an area of $1 \times 3 \mathrm{deg}^{2}$ centred at the Galactic coordinates $l \simeq 1.1, b \simeq-1.7$ aligned with the Galactic plane so that the long axis of the field is parallel to the Galactic plane. We aim to use both the EUCLID optical and near IR channels, although the efficiency is about twice better on the IR channels.

There is also a proposition to extend the initial 3 month additional science program with a 9 month legacy program which would measure the abundance of cool Earth-mass exoplanets around solar like stars. This program (if accepted) could take place once the dark energy objective will have been reached towards the end of the mission. An attractive possibility would be to have a first survey of 3 months shortly after the launch to guarantee early high-profile results (detection of planets down to the mass of Mars). It is also important to have observations early in the mission life time and after few years to maximize the baseline in order to be able to have better constraints on host masses. With the 9 month legacy program in addition to the 3 months that are currently in the additional science program EUCLID could achieve:

a) a complete census of planets down to Earth mass with separations exceeding $1 \mathrm{AU}$

b) complementary coverage to Kepler of the planet discovery space

c) sensitivity to planets down to $0.1 M_{\oplus}$, including all Solar System analogues except for Mercury

d) complete lens solutions for most planet events, allowing direct measurements of the planet and host masses, and distance from the observer.

Currently EUCLID is proposed to ESA as a M-class mission, together with PLATO and SOLAR ORBITER. At most, two out of the three will be selected for launch in 2017-2018. The announcement of the selection process will be made in September 2011. 


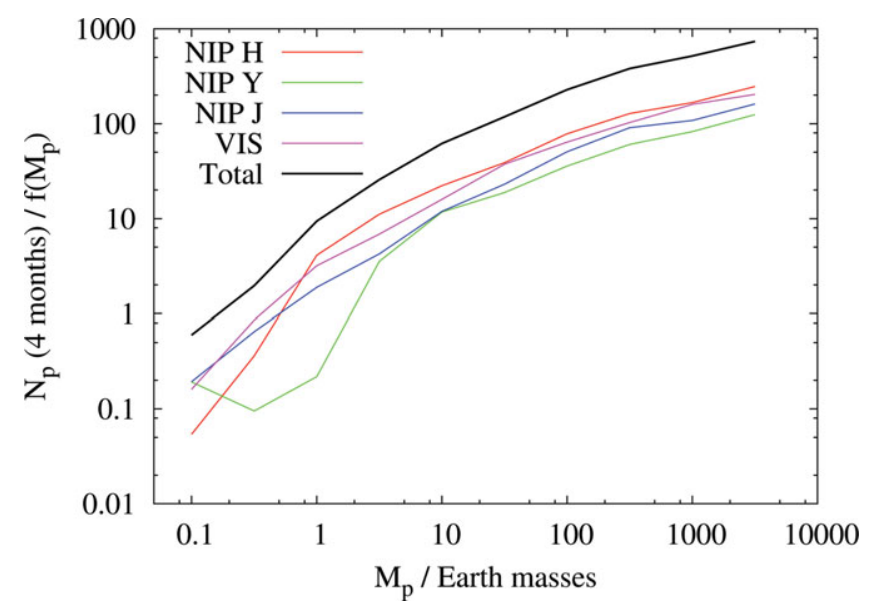

Figure 1. The expected number of planets versus mass for a 4 month program with EUCLID, assuming one planet of mass $M_{p}$ per host and a logarithmic separation prior out to 30AU (so ignoring free-floating planets). The lines show yields for the H, Y and J EUCLID IR channels, as well as the optical channel (VIS) and the overall yield (black line). The planet yield scales roughly linearly with program duration.

Members of the EUCLID exoplanet Working Group are constructing a detailed simulation of the EUCLID exoplanet catch using the Besancon population synthesis model (Robin et al. 2003; Marshall et al. 2006). The model includes bulge, disk and spheroid lens and source populations as well as a detailed prescription for the lens and source kinematics. It allows for specific optical and infrared bandpass predictions and also incorporates a calibrated 3D model of the interstellar dust distribution. This model provides a detailed simulation of the spatial and timescale characteristics of the microlensing events (Kerins, Robin \& Marshall 2009), allowing the EUCLID field placement to be optimized for maximum exoplanet yield. The model is still under development but initial predictions indicate that even a 3-month EUCLID program should provide useful statistics on the planet abundance down to Earth masses (Figure 1).

\subsection{WFIRST}

A Science Definition Team (SDT) of 19 members has been appointed in December 2010. The WFIRST SDT is charged with developing a mission design, but the "straw-man" design used by the Astro2010 Decadal survey, was the JDEM-Omega design (Gehrels 2010), which was one of three mission concepts that served as the basis for the WFIRST concept. The other two mission concepts were the Microlensing Planet Finder (Bennett et al. 2010b) and the Near-Infrared Sky Surveyor (Stern et al. 2010). The "straw-man", JDEM-Omega design uses a $1.5 \mathrm{~m}$ telescope with a wide field-of-view imager that would have three main science programss:

- A microlensing exoplanet survey that would use $\sim 500$ days of the first five years of the mission with a planet yield equal to half that of MPF.

- A dark energy survey using 2-2.5 years of the first five years of the mission employing baryonic acoustic oscillations, weak lensing and Type Ia supernovae.

- Galactic and and extragalactic infra-red surveys.

One of the specificity of the space-based microlensing surveys and the comparison EUCLID-WFIRST is that the planet yield scales roughly linearly with the focal plane 
area and the program duration. Therefore, once a design would have been agreed upon for WFIRST it will be straightforward to have the estimates.

\section{References}

Batista, V., et al. 2009, A\& A, 508, 467

Beaulieu, J. P., et al. 2006, Nature, 439, 437

Beaulieu, J. P., et al. 2008, White Paper submission to the ESA Exo-Planet Roadmap Advisory Team, arXiV:0808.0005

Beaulieu, J. P., et al. 2010, APS Conf. Ser., 430, 266

Bennett, D. P. \& Rhie, S. 1996, ApJ, 472, 660

Bennett, D. P. \& Rhie, S. 2002, ApJ, 574, 985

Bennett, D. P., Anderson, J., \& Gaudi, B. S. 2007a, ApJ, 660, 781

Bennett, D. P., et al. 2007b, White Paper submitted to the NASA/NSF ExoPlanet Task Force, arXiV:0704.0454

Bennett, D. P., et al. 2010a, in "RFI Response to The Astronomy and Astrophysics Decadal Survey", arXiv:1012.4486

Bennett, D. P., et al. 2010b, ApJ, 713, 837

Cassan, A., et al. 2011, submitted

Gaudi, B. S., et al. 2008, Science, 319, 927

Gaudi, B. S., et al. 2009, in "The Astronomy and Astrophysics Decadal Survey", arXiv:0903.0880

Gehrels, N. 2010 in "RFI Response to The Astronomy and Astrophysics Decadal Survey", arXiv:1008.4936v1

Gould, A. \& Loeb, A. 1992, ApJ, 396, 104

Gould, A., et al. 2006, ApJ, 644, L37

Gould, A., Gaudi, B. S., \& Bennett, D. P. 2007, white paper submitted to the NASA/NSF Exoplanet Task Force, arXiv:0704.0767

Gould, A., et al. 2010, ApJ, 720, 1073

Ida, S. \& Lin, D. N. C.. 2005, ApJ, 626, 1045

Kim, S. L., et al. 2010, Proc. SPIE, 7733, 77333

Kerins, E., Robin, A. C., \& Marshall, D. J. 2009, MNRAS, 396, 1202

Lunine, J., et al. 2008, in "Exoplanet Task Force Report", arXiV:0808.2754

Mao, S. \& Paczynski, B. 1991, ApJ, 374, L37

Marshall, D. J., Robin, A. C., Reylé, C., Schultheis, M., \& Picaud, S. 2006, A\& $A, 453,635$

Refregier, A., et al. 2007, "The Dark UNiverse Explorer : proposal to ESA's comic vision", arXiv:0802.2522v4

Robin, A. C., Reylé, C., Derrière, S., \& Picaud, S. 2003, A\&A, 409, 523

Stern, D. 2010, in "RFI Response to The Astronomy and Astrophysics Decadal Survey", arXiv:1008.3563v1

Sumi, T., et al. 2010, ApJ 710, 1641 